\title{
The effect of Monocarboxylate Transporter (MCT1) inhibitor, AR-C117977 on accelerated rejection of cardiac grafts in pre-sensitised rats and concordant xenotransplantation
}

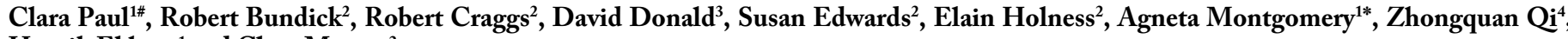 \\ Henrik Ekberg ${ }^{1}$ and Clare Murray ${ }^{2}$ \\ ${ }^{1}$ Department of Surgery, University Hospital, Malmo, Sweden \\ ${ }^{2}$ Department of Discovery BioScience, AstraZeneca R\&D Charnwood, Bakewell Road, Loughborough, Leicestershire LE11 5RH, UK \\ ${ }^{3}$ Department of Medicinal Chemistry AstraZeneca, Charnwood, Loughborough, Leicestershire, UK \\ ${ }^{4}$ Organ Transplantation Institute of Xiamen University, P.R China \\ \#These authors contributed equally to this work
}

\begin{abstract}
Previous studies have demonstrated the immunosuppressive properties of the monocarboxylate transporter MCT1 inhibitor, AR-C117977. The objective of this study was to evaluate the possible limitations in immunosuppressive efficacy of AR-C117977 in models of hyper acute rejection of cardiac transplants in pre-sensitised rats and in concordant cardiac xenotransplantation.

Methods: A primary graft was given without treatment in order to induce sensitisation. At 10 days, a second cardiac transplant was performed, and doses of the test compound were given and compared to or combined with cyclosporin (CsA). Serum samples were retrieved for flow cytometry and measurements of antibody from the presensitised recipients before and after the second transplantation.

Results: Survival was significantly prolonged with AR-C117977 in combination with CsA (median 12.5 days) compared with single treatment. AR-C117977 had a better effect on antibody formation and binding to B-cells than CsA, but a similar effect on T-cells. Acute rejection could not be prevented in any of the treatment groups in the xenotransplant model.

Conclusion: A short course of the MCT1 inhibitor AR-C117977, in combination with CsA, prevented accelerated acute rejection in presensitised rats, but no longterm graft survival was achieved. Rejection in xenotransplantation was not prevented.
\end{abstract}

\section{Introduction}

For many years, the importance of antibody formation in chronic rejection and how to prevent chronic rejection has been discussed widely [1-3]. Half a century ago, acute rejection was the main problem in transplantation and there were only a few immunosuppressive therapies available. As a result, many patients lost their grafts. Today, the main problem is to detect and prevent chronic rejection. Recipients with donor specific antibodies are in need of high immunosuppressive regimes to prevent graft loss. The calcineurin inhibitors (CNI), such as CsA and tacrolimus, are nephrotoxic and in high doses detrimental to kidneys. However, combinations of different drugs in moderate doses would theoretically potentiate the immunosuppressive effect by blocking multiple pathways. Most centres worldwide use a cocktail of different drugs at lower doses to minimize side-effects of the immunosuppressive drugs but with sufficient efficacy to prevent acute rejection. But there is still no drug on the market efficient enough to solve chronic rejection and formation of antibodies.

In the situation of hyper acute rejection, there are both a massive cellular and a humoral component. To combine different immunosuppressive drugs with different immunomodulatory mechanisms could be one way of blocking different immunological pathways and reducing loss of grafts in chronic rejection. In transplantation, a major issue is to prevent acute and chronic rejection without the need for immunosuppressive agents with toxic side-effects.

Monocarboxylate transporters (MCTs) are interesting targets for inhibition of the immune system. The MCTs have numerous physiological functions as they are expressed in a wide range of tissues. Mainly, they control the transport of monocarboxylates such as lactate across the cell membrane. They play a functional role in T-cell

Correspondence to: Clara Paul, Agneta Montgomery, Department of Surgery, Institution of Surgical Science, Malmö, Lund University, Sweden, Email: helenclarapaul@gmail.com

Key words: immunosuppression, monocarboxylate transporter, allograft, antibody, xenotransplantation, presensitised

Received: November 03, 2017; Accepted: November 27, 2017; Published: November 30, 2017 
activation and by inhibiting the MCT1 transporter, the immunological response is reduced [4].

By inhibiting MCT1, we could induce and maintain long-term graft survival in both heart and non-vascularised transplantation models in rat with limited signs of toxicity [4-8]. The rejection is initiated by preexisting humoral immunity and manifests minutes after transplantation. If the graft is left implanted, the preexisting antibodies immediately adhere to the implant and a massive systemic inflammatory response is initiated to destroy the tissue of the graft.

In our previous studies, AR-C117977, a representative member of the class of MCT1 inhibitors, significantly prolonged cardiac allograft survival in rodents with induction of operational tolerance and prevention of graft vasculopathy. In non-vascularised models, AR-C117977 induced long-term skin graft survival and significantly prolonged islet graft survival with monotherapy or in combination with CsA, respectively $[5,6]$.

To challenge the immune response, two different models with preformed antibodies against donors were used to create hyper acute rejection models. To create a condition for hyper acute rejection, antibody formation was induced with two subsequent transplantations in the same recipient. The first was without immunosuppressive treatment leading to rejection and a second transplantation was then performed, with a graft from the same donor strain, but with compound treatment. In xenotransplantation the recipient had innate immunity and preformed antibodies against the donor [9].

The objective of this study was to evaluate graft survival and the possible limitations in immunosuppressive efficacy of AR-C117977 in different accelerated acute rejection models of cardiac transplants in presensitised rats and in concordant cardiac xenotransplantation.

\section{Material and methods}

\section{Animals}

For the in vitro and in vivo murine antibody studies, Balb/c mice were obtained from Harlan, UK and experiments were performed in accordance with UK Home Office regulations. Isogenic rats and mice were obtained from Mollegaard Breeding \& Research Centre Ltd (Denmark) and Syrian hamsters from Charles River, Germany. Animals were conditioned for at least one week prior to transplantation at our laboratory. They were housed in standard cages under controlled light/dark cycles, and fed standard laboratory diet with free access to water. Male rats of the strains DA $\left(\mathrm{RT}^{\mathrm{avl}}\right)$ and PVG $\left(\mathrm{RT}^{\mathrm{c}}{ }^{\mathrm{c}}\right)$ were used. Recipients weighed 180-240 g and donors 100-160 g. Syrian hamsters $(80-100 \mathrm{~g})$ and $\mathrm{BALB} / \mathrm{C}$ mice $(20 \mathrm{~g})$ were used as donors in the xenotransplant experiments and PVG rats as recipients. The study design was approved by the Research Ethics Committee of Lund University, and all procedures were performed in accordance with the Good Laboratory Practice code published by the National Board of Health and Welfare in Sweden.

\section{Inhibition of murine B-lymphocyte proliferation and antibody production in vitro}

Splenic B-lymphocytes were obtained from Balb/c mice by disruption of the spleen through a nylon sieve. The resultant cellular suspension was washed three times by centrifugation. The spleen cells were plated out in flat bottomed 96 well microtitre plates at a concentration of $3 \times 10^{6}$ cells per well. B-lymphocyte stimulators were added with or without compound. Stimulators used were lipopolysaccharide (LPS) at $2 \mu \mathrm{g} / \mathrm{ml}$ and 8 -mercaptoguanosine (8MG) at $100 \mu \mathrm{g} / \mathrm{ml}$. LPS and $8 \mathrm{MG}$ were both obtained from Sigma (Poole, Dorset, UK). For proliferation assays, the cultures were incubated for $48 \mathrm{~h}$ at $37^{\circ} \mathrm{C}$ and pulsed with $0.5 \mu \mathrm{Ci}$ tritiated thymidine (GE Healthcare, Amersham, UK) for the final $6 \mathrm{~h}$ of culture. The cells were harvested onto glass fibre filter mats and radioactivity incorporated due to thymidine uptake used as a measure of proliferation. For antibody production, the cultures were incubated for $96 \mathrm{~h}$ at $37^{\circ} \mathrm{C}$ and the supernatants were assayed for total Ig by ELISA.

\section{Inhibition of murine antibody production in vivo}

To measure effects of compound on IgG2a production, Balb/c mice were immunised with $5 \mathrm{mg} / \mathrm{kg}$ ovalbumin (OVA, Sigma, Poole, UK) in poly I:C adjuvant (polyinosinic:polycytidylic acid adjuvant). After 14 days the mice were re-challenged with ovalbumin in buffered saline vehicle and 7 days later serum samples were taken and analysed for total and specific IgG2a. Compound was dosed daily by the subcutaneous route from re-challenge to termination prior to serum collection. For total IgG2a, microtitre plates were coated with $5 \mu \mathrm{g} / \mathrm{ml}$ of goat antimouse IgG2a in PBS and incubated overnight at $4^{\circ} \mathrm{C}$. The plates were then washed four times with PBS containing $0.05 \%$ Tween 20 and blocked with $1 \%$ BSA at room temperature for $2 \mathrm{~h}$. This was followed by two further plate washes. The serum samples and IgG2a standards were added to the wells in duplicate and incubated at $4^{\circ} \mathrm{C}$ overnight. The plates were then washed a further four times before adding $50 \mu \mathrm{l}$ alkaline phosphatase conjugated goat anti-mouse IgG diluted in $0.1 \%$ BSA in PBS and left at room temperature for $1 \mathrm{~h}$. The plate was washed a further four times before enzyme substrate $(1 \mathrm{mg} / \mathrm{ml}$ p-nitrophenyl phosphate in $1 \mathrm{M}$ diethanolamine buffer $\mathrm{pH} 9.8$ ) at $1 \mathrm{mg} / \mathrm{ml}$ was added. The colorimetric reaction was detected at $405 \mathrm{~nm}$ in a plate reader.

For specific IgG2a, the methodology was similar except for the following. The microtitre plates were coated with $50 \mu \mathrm{g} / \mathrm{ml} \mathrm{OVA.} \mathrm{The}$ serum samples or normal control sera were added to the washed plates. Alkaline phosphatase conjugated goat anti-mouse IgG2a was used as the detection reagent.

\section{Heart transplantation}

The donor and recipient operations were performed under clean but not sterile conditions. The rats were anaesthetized with intraperitoneal (i.p.) injection of Dormicum ${ }^{\circledR}$ and Hypnorm ${ }^{\circledR}$.The donor heart was flushed with cold Perfadex ${ }^{\circledR}$ (Pharmacia \& Upjohn, Lund, Sweden), and the caval and pulmonary veins were ligated before removal. The first heart was heterotopically transplanted to the right neck vessels of the recipient, the aortic root being anastomosed to the common carotid artery and the pulmonary artery to the jugular vein with non-suture cuff technique [10]. In retransplants, 10 days after the first transplantation, the grafts were placed in the groin and anastomosed to the femoral vessels. Allograft function was monitored twice daily by palpation, rejection being defined as the cessation of a palpable heartbeat. The xenotransplants were performed as described above with the graft transplanted to the right neck vessels of the recipient.

\section{Compounds}

AR-C117977 was synthesised in the Department of Medicinal Chemistry, AstraZeneca R\&D Charnwood and was supplied in powder form. Suspensions of the compound in 5\% tween $20(\mathrm{v} / \mathrm{v})$ and $0.6 \%$ carboxymethyl cellulose solution were prepared and stored at $-20^{\circ} \mathrm{C}$. Suspensions of three different concentrations were prepared; $10 \mathrm{mg} /$ $\mathrm{ml}, 30 \mathrm{mg} / \mathrm{ml}$ and $100 \mathrm{mg} / \mathrm{ml}$. The suspensions were mixed thoroughly before administration. Subcutaneous (s.c.) injections were performed once daily from day 0 to 9 and a volume of $0.2 \mathrm{ml}$ of a specific 
Paul C (2017) The effect of Monocarboxylate Transporter (MCT1) inhibitor, AR-C117977 on accelerated rejection of cardiac grafts in pre-sensitised rats and concordant xenotransplantation

suspension was injected in rats weighing $200 \mathrm{~g}$ to reach the target dose. Cyclosporine A (CsA, Sandimmun ${ }^{\circledR} 100 \mathrm{mg} / \mathrm{ml}$; Novartis, Basle, Switzerland) was dissolved in Intralipid ${ }^{\circledR}(200 \mathrm{mg} / \mathrm{ml}$, Pharmacia \& Upjohn, Lund, Sweden) to a final concentration of $4 \mathrm{mg} / \mathrm{ml}$ and given orally for ten days [11]. A volume of $0.5 \mathrm{ml}$ was given once daily to rats weighing $200 \mathrm{~g}$. CsA was given immediately pre-operatively and ARC117977 three hours before transplantation.

\section{Groups}

Sensitization in rats was induced by a primary cardiac graft left untreated for 10 days and then a secondary graft was transplanted (at day 0$)$. CsA (10 mg/kg orally), AR-C117977 (10 mg/kg, $30 \mathrm{mg} / \mathrm{kg}$ or $100 \mathrm{mg} / \mathrm{kg}$ by once daily subcutaneous injection) or the combined treatment of CsA and AR-C117977 was given day 0-9. In mouse to rat cardiac transplants, either CsA, AR-C117977 $(30 \mathrm{mg} / \mathrm{kg})$ or the combined treatment was given. Hamster to rat transplant recipients were given the high dose of AR-C117977 (100 mg/kg s.c. inj.). Four pre-treatment groups were included with compounds given from three days before transplantation onwards. In all protocols, one group with no treatment was used as control.

\section{Flow cytometry analysis}

For T- and B-cell proliferation assays, antibodies were purchased from AbDSerotec, Oxford, U.K. and R\&D Systems, Minneapolis, USA. Mouse anti-rat CD3:FITC and CD45RA:RPE was used primarily to stain for T-cells and B-cells respectively. A second antibody goat antirat IgG-APC, was used as a binding reagent to the preformed antibodies for immunofluorescent staining.

From the rat recipients, serum samples were taken before the first transplantation (day-10). Just before and after the second transplantation, in these presensitised rats, serum samples for donorspecific antibodies were retrieved at day $0,7,14$ and 21. Splenocytes were prepared from the spleen of the same rat strain as the donor
(DA) under sterile conditions. The splenocytes were washed twice with Isoflow and incubated for 10 minutes with endogenous serum. After washing, the splenocytes were incubated for 10 minutes with exogenous serum from the recipient rats. Finally, after washing with Isoflow, the cells were incubated with anti-CD3, anti-CD45 and anti-IgG for 10 minutes. One $\mathrm{ml}$ of Fix-and-lyse solution was added to the samples and thereafter additionally incubated for 10 minutes. Thereafter, the cells were analysed by flow cytometry (FC 500 flow cytometer; BeckmanCoulter).

\section{Statistical analysis}

Graft survival was plotted according to Kaplan-Meier and differences between groups were compared using log-rank statistics.

\section{Results}

The effects of the MCT inhibitor on B-lymphocyte function were investigated in in vitro and in vivo models of antibody production. Firstly, splenocytes from Balb/c mice were stimulated with LPS or $8 \mathrm{MG}$ to induce proliferation and antibody production (Figure 1), AR-C117977 and CsA inhibited proliferation in response to both stimuli (Figure 1A and 1B). Although the maximum inhibition with AR-C117977 (46\%) was lower than the full inhibition observed with CsA, the potency of the MCT inhibitor was approximately 100-fold higher (IC50 1-2 nM for AR-C117977 vs IC50 $370 \mathrm{nM}$ for CsA). In addition to inhibition of proliferation, AR-C117977 and CsA inhibited antibody production induced by both LPS and 8MG (Figure 1C and 1D). AR-C117977 showed approximately $70 \%$ inhibition of antibody production and was 100-fold more potent than CsA (IC50 0.5-2 nM vs 200-400 nM for CsA). The ability of AR-C117977 to reduce antibody production was confirmed in an in vivo model of OVA and polyI:Cstimulated IgG2a production in Balb/c mice (Figure 2). AR-C117977 showed $57 \%$ inhibition of total IgG2a and $74 \%$ inhibition of OVAspecific IgG2a at the $30 \mathrm{mg} / \mathrm{kg}$ dose.
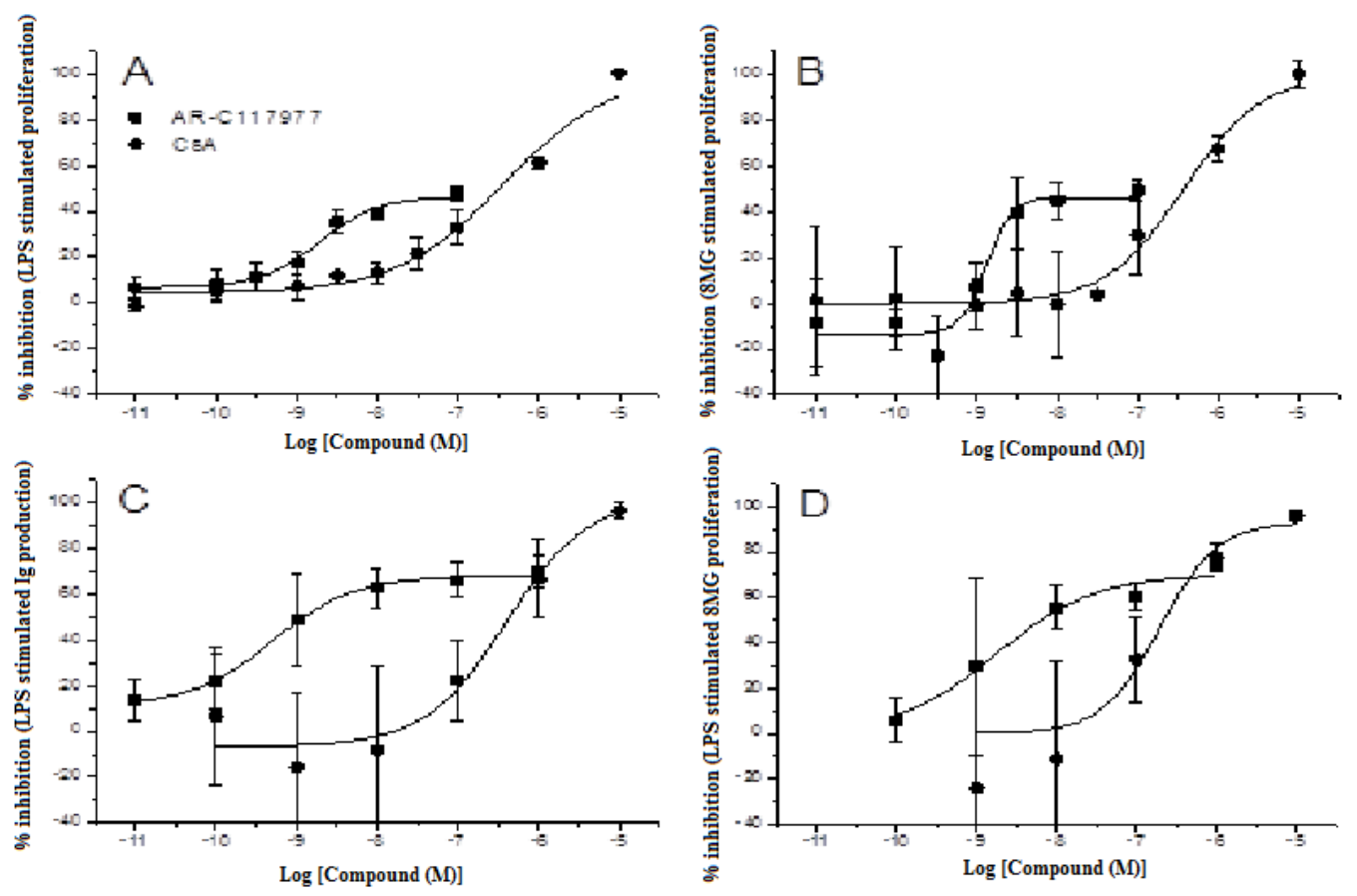

Figure 1. Inhibition of murine splenocyte proliferation and antibody production. Proliferation of murine Balb/c splenocytes was measured $48 \mathrm{~h}$ after stimulation with $2 \mu \mathrm{g} / \mathrm{ml} \mathrm{LPS}$ (A) or $100 \mu \mathrm{g} / \mathrm{ml} 8 \mathrm{MG}$ (B) in the presence of AR-C117977 or CsA. The effect of compounds on splenocyte antibody production was measured by ELISA $96 \mathrm{~h}$ after stimulation with LPS (C) or $8 \mathrm{MG}$ (D). 
Paul C (2017) The effect of Monocarboxylate Transporter (MCT1) inhibitor, AR-C117977 on accelerated rejection of cardiac grafts in pre-sensitised rats and concordant xenotransplantation

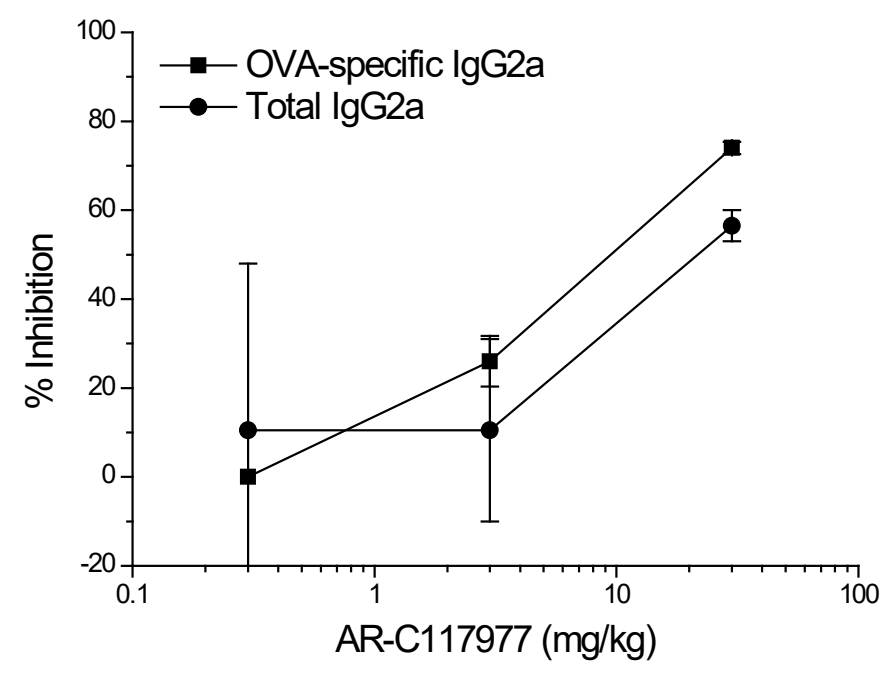

Figure 2. Inhibition of murine antibody production in vivo $\mathrm{Balb} / \mathrm{c}$ mice were sensitised with OVA and polyI:C, challenged with OVA at day 14 and total and specific IgG2a were measured by ELISA in serum samples collected at day 21. AR-C117977 was dosed s.c. daily for the last 7 days prior to serum collection. Data shown are the mean of two independent experiments.

In pre-sensitised rats, primary allograft survival in untreated rats (groups 1-7; Table 1) ranged from 6-8 days. Survival of the second cardiac graft in the sensitized rats was limited to 1 day without treatment and 2 days with AR-C117977 at doses of $10-100 \mathrm{mg} / \mathrm{kg}$. CsA prolonged graft survival for a median of 6 days, but all transplants rejected during treatment (which ceased on day 9). With combined therapy of CsA and AR-C117977, graft survival was significantly prolonged to a median of 11.5 and 12.5 days, respectively, with doses of AR-C117977 being $10 \mathrm{mg} / \mathrm{kg}$ and $30 \mathrm{mg} / \mathrm{kg}(\mathrm{P}=0.0005)$. In cardiac xenotransplants from mouse $(\mathrm{BALB} / \mathrm{C})$ to rat an early acute rejection occurred at a range of 4-7 days after transplantation. It was seen in all groups of AR-C117977, CsA or during combined treatment (Table 2). Likewise, in hamster to rat transplants, early acute rejection occurred at 2-4 days in all treatment groups, including those given combined pretreatment for three days before transplantation (Table 3).

In flow cytometry, binding of antibodies to lymphocytes was measured. Sera from the presensitised rats in different dose and treatment groups were added to donor splenocytes. Compared to the non-treatment group, sera from animals receiving treatment with CsA, AR-C117977 or a combination of the two showed less binding of antibodies to T-cells compared to the group without any treatment. In both the non-treatment and the CsA treated groups the antibody response to B-cells was higher than in groups treated with the test drug. Thus, AR-C117977 had a better immunosuppressive effect on antibody binding to donor B-cells than CsA but similar effect on T-cells (Figure $3 \mathrm{~A}$ and $3 \mathrm{~B})$.

\section{Discussion}

The focus of this study was to understand the effect of AR-C117977 on antibody production and to investigate whether AR-C117977 could induce donor-specific suppression in animal models with preformed antibodies and prevent hyper acute rejection.

It is well known that preformed antibodies against the donor mediates hyper acute rejection. Patients who develop antibodies against the donor are a greater challenge considering treatment strategies and survey than patients without preformed antibodies. Our group has
Table 1. Prevention of accelerated rejection in pre-sensitised recipients of cardiac allografts in DA to PVG rats with treatment started the same day (day 0). First transplantation was performed on day -10 , and rats were given no immunosuppressive treatment for 10 days until day 0 . The second transplantation was performed on day 0 . Treatment of AR-C117977 was given for ten days (0-9) and dosed $10,30 \mathrm{mg} / \mathrm{kg}$ (s.c. inj.), or $100 \mathrm{mg} / \mathrm{kg}$ (orally). Treatment of CsA days $0-9$ was given orally in a dose of $10 \mathrm{mg} / \mathrm{kg}$.

\begin{tabular}{|c|c|c|c|c|}
\hline $\begin{array}{l}\text { Groups } \\
\text { (treatment) }\end{array}$ & $\begin{array}{l}\text { First graft } \\
\text { survival } \\
\text { (days) tx day } \\
-10\end{array}$ & $\begin{array}{l}\text { Median } \\
\text { first graft } \\
\text { survival } \\
\text { (days) }\end{array}$ & $\begin{array}{l}\text { Second graft } \\
\text { survival (days) } \\
\text { tx day } 0\end{array}$ & $\begin{array}{l}\text { Median } \\
\text { second graft } \\
\text { survival } \\
\text { (days) }\end{array}$ \\
\hline 1. No treatment & $6 \quad 6 \quad 7 \quad 7 \quad 78$ & 7 & $\begin{array}{lllllll}1 & 1 & 1 & 1 & 1 & 1\end{array}$ & 1 \\
\hline \multicolumn{5}{|l|}{ CsA } \\
\hline $\begin{array}{l}\text { 2. CsA (10 mg/ } \\
\mathrm{kg} \text { ) }\end{array}$ & $\begin{array}{llll}6 & 6.5 & 6.5 & 6.5 \\
7 & 7 & & \end{array}$ & 6.5 & $\begin{array}{llllll}4 & 6 & 6 & 6 & 7 & 8\end{array}$ & 6 \\
\hline \multicolumn{5}{|l|}{ AR-C117977 } \\
\hline $\begin{array}{l}\text { 3. AR-C117977 } \\
(10 \mathrm{mg} / \mathrm{kg})\end{array}$ & $\begin{array}{llllll}6 & 6 & 7 & 7 & 7 & 8\end{array}$ & 7 & $\begin{array}{lllll}1.5 & 1.5 & 2 & 2 \\
2 & 2 & & & \end{array}$ & 2 \\
\hline $\begin{array}{l}\text { 4. AR-C117977 } \\
(30 \mathrm{mg} / \mathrm{kg})\end{array}$ & $\begin{array}{llllll}6 & 6 & 7 & 7 & 8 & 8\end{array}$ & 7 & 1.52222222 & 2 \\
\hline $\begin{array}{l}\text { 5. AR-C117977 } \\
(100 \mathrm{mg} / \mathrm{kg})\end{array}$ & 6777 & 7 & $\begin{array}{llll}2 & 2 & 2.5 & 2.5\end{array}$ & 2.25 \\
\hline \multicolumn{5}{|c|}{ AR-C117977 + CsA } \\
\hline $\begin{array}{l}\text { 6. CsA }(10 \mathrm{mg} / \\
\mathrm{kg})+ \\
\text { AR-C117977 } \\
(10 \mathrm{mg} / \mathrm{kg})\end{array}$ & $\begin{array}{llll}6 & 6 & 6 & 6.5 \\
6.5 & 7 & \end{array}$ & 6.25 & $\begin{array}{llll}9 & 9 & 11 & 12 \\
14 & 17 & \end{array}$ & $11.5 * * *$ \\
\hline $\begin{array}{l}\text { 7. CsA }(10 \mathrm{mg} / \\
\mathrm{kg})+ \\
\text { AR-C117977 } \\
(30 \mathrm{mg} / \mathrm{kg})\end{array}$ & $\begin{array}{llllll}6 & 6 & 7 & 7 & 8 & 8\end{array}$ & 7 & $\begin{array}{llll}9 & 9 & 12 & 13 \\
15 & 18 & \end{array}$ & $12.5 * * *$ \\
\hline
\end{tabular}

Table 2. Graft survival of mouse (BALB/C) to rat (PVG) cardiac xenotransplantation Cyclosporine (CsA, orally) and/or ARC-117977 (subcutaneous injections) were given from the day of transplantation and for 10 days.

\begin{tabular}{|c|c|}
\hline Groups (treatment) & Graft survival (days) \\
\hline 1. No treatment & 445 \\
\hline \multicolumn{2}{|l|}{ CsA } \\
\hline 2. CsA (10 mg/kg) & 466 \\
\hline \multicolumn{2}{|l|}{ AR-C117977 } \\
\hline 3. AR-C117977 (30 mg/kg) & 567 \\
\hline \multicolumn{2}{|l|}{ CsA + AR-C117977 } \\
\hline 4. CsA $(10 \mathrm{mg} / \mathrm{kg})+\mathrm{AR}-\mathrm{C} 117$ & 444.5 \\
\hline
\end{tabular}

Table 3. Graft survival of hamster to rat (PVG) cardiac xenotransplantation. Cyclosporine (CsA, orally) and/or ARC-117977 (subcutaneous injections) were given from the day of transplantation and for 10 days. In groups 5-8, treatment was started 3 days before transplantation.

\begin{tabular}{|c|c|}
\hline Groups (treatment) & Graft survival (days) \\
\hline 1. No treatment & 22.53 \\
\hline \multicolumn{2}{|l|}{ CsA } \\
\hline 2. CsA (10 mg/kg) & 22.52 .5 \\
\hline \multicolumn{2}{|l|}{ ARC-117977 } \\
\hline 3. ARC-117977 (30 mg/kg) & 333 \\
\hline 4. ARC-117977 (100 mg/kg) & 333 \\
\hline \multicolumn{2}{|l|}{ CsA + ARC-117977 } \\
\hline $\begin{array}{l}\text { 4. CsA (10 mg/kg) + ARC-117977 (100 } \\
\mathrm{mg} / \mathrm{kg})\end{array}$ & 333 \\
\hline \multicolumn{2}{|l|}{ Pretreatment from day -3} \\
\hline 5. CsA $(10 \mathrm{mg} / \mathrm{kg})$ & 222.5 \\
\hline \multicolumn{2}{|l|}{ ARC-117977 } \\
\hline 6. ARC-117977 (30 mg/kg) & 2.533 \\
\hline 7. ARC-117977 (100 mg/kg) & 2.534 \\
\hline \multicolumn{2}{|l|}{ CsA + ARC-117977 } \\
\hline $\begin{array}{l}\text { 8. CsA }(10 \mathrm{mg} / \mathrm{kg} \text { oral })+\mathrm{ARC}-117977 \\
(100 \mathrm{mg} / \mathrm{kg})\end{array}$ & 2.533 .5 \\
\hline
\end{tabular}


Paul C (2017) The effect of Monocarboxylate Transporter (MCT1) inhibitor, AR-C117977 on accelerated rejection of cardiac grafts in pre-sensitised rats and concordant xenotransplantation
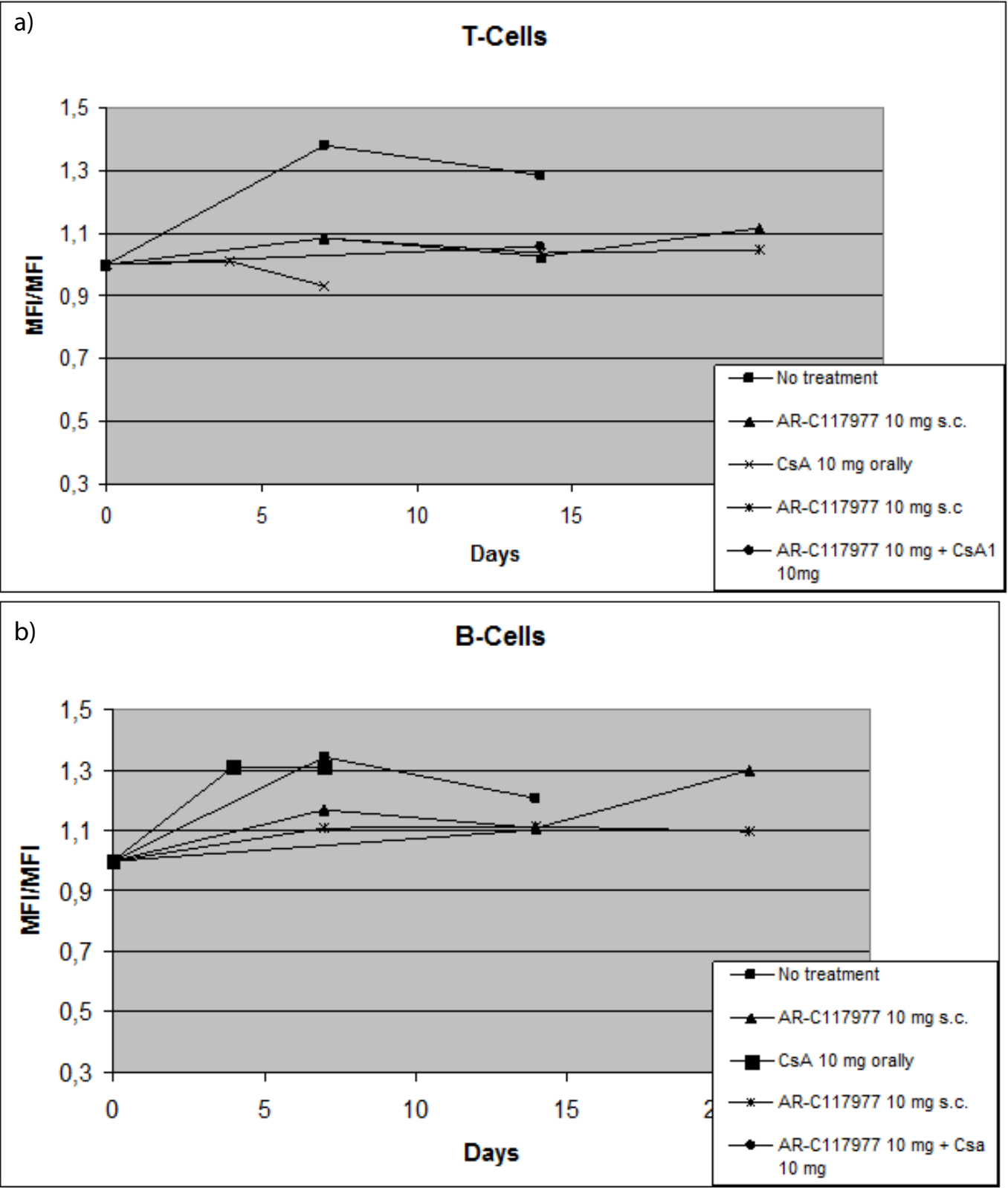

Figure 3A and 3B. AR-C117977 and suppression of antibody formation against donor lymphocytes.

Sera from presensitised rat recipients (PVG), taken at day $0,7,14,21$ were added to donor (DA) lymphocytes. Binding of antibodies to lymphocytes was measured and compared between treatment groups with flow cytometry. Because of the variation between MFI in all animals, T- and B-cell ratio was calculated to easier see the tendencies between treatment groups.

previously done several studies with heart transplantation in rodents and has established experience of the method. In this study, our first choice of method was heart transplantation. To continue to explore the immunosuppressive properties of AR-C117977, we set up hyper acute rejection xeno- and allograft heart models in rat to challenge the efficacy of the compound and to explore development and inhibition of de novo antibodies. In the allograft model, the recipients were conditioned for hyper acute rejection by two consecutive transplantations.

We also wanted to investigate whether there was a difference in the effect of the compound between de novo antibodies, as in the allograft model, and preformed antibodies against species.

The MCT1 inhibitor AR-C117977, in combination with CsA, prevented accelerated acute rejection in presensitised rats. However, no long-term graft survival was achieved. Rejection treatment was ineffective in the xenotransplant model.

AR-C117977, inhibited murine splenocyte proliferation in vitro and antibody production both in vitro and in vivo. These data suggest that activated B-lymphocytes have a high rate of aerobic glycolysis leading to lactate production such that inhibition of lactate efflux by MCT1 blockade reduces the ability of the cells to proliferate and produce antibodies. The dependence of activated T-lymphocytes on aerobic glycolysis (the Warburg effect) has now been established [12] but the metabolic processes required for B-lymphocyte activation and function have not been widely investigated although increased glucose metabolism after activation has been shown [13]. Our study provides evidence that MCT blockade can affect the function of B-lymphocytes 
Paul C (2017) The effect of Monocarboxylate Transporter (MCT1) inhibitor, AR-C117977 on accelerated rejection of cardiac grafts in pre-sensitised rats and concordant xenotransplantation

in vitro and in vivo in addition to causing immunosuppression via inhibition of T-lymphocyte proliferation as we have previously described [4]. These data are consistent with a previous report of the effect of an MCT inhibitor on rat B-lymphocytes in vitro [14]. Earlier experiments demonstrated that the MCT-1 inhibitor, AR-C117977, significantly prolongs allograft survival, prevents chronic rejection and induces tolerance in the rat cardiac and skin allograft models.

In the allograft model, second graft survival was significantly prolonged in the group treated with CsA but by blocking MCT1 alone, the graft survival was poor. However, the combination of AR-C117977 with CsA significantly potentiated the prevention of accelerated acute rejection. One could speculate whether this is due to the massive proliferation of lymphocytes and the insufficient response when blocking MCT1 alone and that suppression must cover several possible immunological pathways. Like in stimulated T-cells, cancer cells proliferate at a high rate and produce lactate as a result of aerobic glycolysis. Cancer cells adapt to a nutrient-poor environment and can up-regulate enzymes and other molecules in the cell to promote malignant progression of the cancer [15]. Blocking MCT1 selectively in a powerful T-cell response could trigger an up-regulation of other transporters to transport lactic acid out of the cell or cause a switch to other metabolic pathways for energy production such as glutaminolysis.

In this study we wanted to investigate if AR-C117977 alone or in combination with CsA had an effect on cellular and humoral responses and, if so, MCT1 inhibition could be a contribution to the treatment used in clinic today.

In the xenotransplant models, we saw no effect on graft survival between control and treatment groups. Unfortunately, at the time, we did not collect sera samples from rats transplanted with xenografts because of the poor survival rate. In the allograft model, we saw an effect on suppression of antibody formation in the treatment groups in the flowcytometry analysis. Despite the poor outcome of the xenografts it would have been interesting to see if there was an effect of AR-C117977 on the antibody response. Little is known about differences between antibodies already preformed in xenotransplantation compared with de novo antibodies formed in an allograft transplantation setting. It might be a difference in mechanisms or grade of immune response of between these different models and this is to be elucidated.

In summary, we previously demonstrated evidence of prolonged allograft survival, prevention of chronic rejection and induction of donor-specific tolerance in rat cardiac and skin allograft models. We have now defined the limit of efficacy of AR-C117977 and demonstrated that MCT1 inhibition was unable to prevent hyperacute rejection in models with preformed antibodies.

\section{Conflict of interest}

The study was supported by unrestricted educational grant from AstraZeneca, Charnwood.

\section{References}

1. Blume O, Yost S (2012) Antibody-Mediated Rejection:Pathogenesis, Prevention, Treatment, and Outcomes. Journal of Transplantation 201754.

2. Ho E, Vlad G (2011) Pre- and posttransplantationallosensitization in heart allograft recipients: Major impact of de novo alloantibody production on allograft survival. Human Immunology 72: 5-10. [Crossref]

3. Costello JP, Mohanakumar T, Nath DS (2013) Mechanisms of chronic cardiac allograft rejection. Tex Heart Inst J 40: 395-399. [Crossref]

4. Murray CM, Hutchinson R, Bantick JR, Belfield G, Benjamin AD, et al. (2005) Monocarboxylate transporter MCT1 is a target for immunosuppression. Nature Chemical Biology 1: 371.

5. Ekberg H, Qi Z, Påhlman (2007) The specific monocarboxylate transporter-1 (MCT1) inhibitor, AR-C 117977, reduces acute and chronic allograft rejection and induces operational tolerance in the rat. Transplantation. 15: 84(9): 1191-1199. [Crossref]

6. Clara Påhlman, Helene Malm, Zhongquan Qi (2008) Operational tolerance in non-vascularised transplant models induced by AR-C117977, a monocarboxylate transporter (MCT-1) inhibitor. Transplantation. 86(8): 1135-1138.

7. Påhlman C, Qi Z, Murray CM, Ferguson D (2013) Immunosuppressive properties of a series of novel inhibitors of the monocarboxylate transporter MCT-1. Transpl Int 28(1): 22-29. [Crossref]

8. Baltazar F, Pinheiro C, Morais-Santos F (2014) Monocarboxylate transporters as targets and mediators in cancer therapy response. Histol Histopathol 29(12): 15111524. [Crossref]

9. Li S, Waer M, Billiau AD (2009) Xenotransplantation: role of natural immunity Transpl Immunol 21: 70-74. [Crossref]

10. Olausson M, Mjörnstedt L, Lindholm L, Brynger H (1984) Non-suture organ grafting to the neck vessels in rats. Acta Chir Scand 150: 463-467. [Crossref]

11. Lim SM, White DJ (1989) Cyclosporin A and tolerance induction in experimental animals. In: Thomson AW (ed.) Cyclosporin: mode of action and clinical application. Boston: Kluwer Academic Publishers: 82.

12. Wang R, Green DR (2012) The immune diet: meeting the metabolic demands of lymphocyte activation. F1000 Biology Reports 4: 9. [Crossref]

13. Doughty CA, Bleiman BF, Wagner DJ (2006) Antigen receptor-mediated changes in glucose metabolism in B lymphocytes: role of phosphatidylinositol 3-kinase signalling in the glycolytic control of growth. Blood 107 (11): 4458-4465. [Crossref]

14. Cho KS, Yamada T, Wynn C (2010) Mechanism Analysis of Long-Term Graft Survival by Monocarboxylate Transporter-1 Inhibition Transplantation 90 (12): 1299-1306. [Crossref]

15. Pinheiro C, Longatto-Filho A, Azevedo-Silva J, Casal M, Schmitt FC, et al. (2012) Role of monocarboxylate transporters in human cancers: state of the art. $J$ Bioenerg Biomembr 44: 127-139. [Crossref]

Copyright: (C2017 Paul C. This is an open-access article distributed under the terms of the Creative Commons Attribution License, which permits unrestricted use, distribution, and reproduction in any medium, provided the original author and source are credited. 